\title{
EIGER2 CdTe detectors for hard X-ray research under extreme conditions
}

\author{
T. Donath, M. Burian, S. Brandstetter
}

DECTRIS Ltd, 5405 Baden-Dättwil, Switzerland

tilman.donath@dectris.com

In the last decade, Hybrid Photon Counting (HPC) X-ray detectors [1] like the PILATUS have transformed synchrotron research. They provide noise-free detection and enable new data acquisition modes. The most current HPC detector family EIGER2 enables even more ambitious X-ray science. These detectors combine all advantages of previous generations while offering new acquisition features and improved performance: maximum count rates of $10^{7}$ photons/sec per pixel, small pixels of $75 \mu \mathrm{m} \times 75 \mu \mathrm{m}$, two energydiscriminating thresholds, and frame rates up to $2 \mathrm{kHz}$ with zero dead time $(<100 \mathrm{~ns})$ between exposures.

EIGER2 detectors were designed and optimized for the demands of synchrotron applications, and they are available for the laboratory as well. Equipped with CdTe sensors they provide high quantum efficiency at energies up to $100 \mathrm{keV}$, making them ideal for hard xray diffraction applications. Two energy thresholds allow for reduction of high-energy background such as from cosmic radiation, higher harmonics, or unwanted sample fluorescence. These benefits advance established X-ray diffraction methods in general like crystallography including powder diffraction as well as scattering techniques. Fast and gated measurements become possible and empower new fields of research, by enabling e.g., time-resolved or pump-probe techniques such as in laser-heating or fast compression and decompression experiments [2].

We will demonstrate the advantages of the HPC CdTe technology for high-pressure X-ray research. We show results from characterization and application measurements carried out in the laboratory and at synchrotron beamlines (ESRF, DLS, BSRF, APS) using loan detectors and the recently installed EIGER2 CdTe systems.

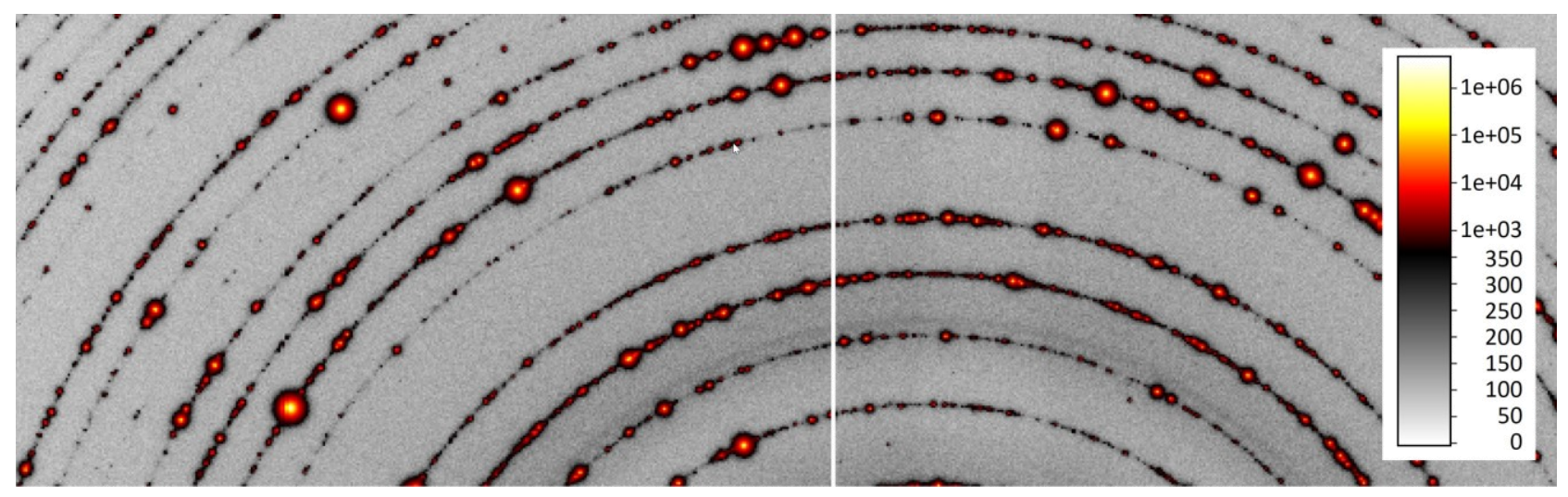

Figure 1. Powder data collected with an EIGER2 X CdTe system at the high-pressure beamline ID27 of the ESRF

[1] Förster, A. et al. (2019), Philos Trans R Soc Math Phys Eng Sci, 377, 20180241

[2] Shen, G et al. (2017), Rep. Prog. Phys. 80, 016101

Keywords: High-pressure research, photon-counting x-ray detector, cadmium telluride, powder diffraction, time-resolved 